\title{
A NOTE ON TAUBERIAN THEOREMS FOR REGULARLY GENERATED SEQUENCES
}

\author{
İBRAHIMM C̣ANAK AND ÜMİT TOTUR
}

\begin{abstract}
We prove some Tauberian theorems which generalize results in [5, Theorems 1 and 2] and [4, Theorem $3.2 .2]$
\end{abstract}

\section{Introduction}

Let $(A)$ denote the Abel method of summability of a sequence $\left(s_{n}\right)$ of real numbers and $\mathscr{S}$ the class of slowly oscillating sequences in the sense of Stanojevic [7]. Canak [1] proved the following theorem known as the generalized Littlewood tauberian theorem [6].

Theorem 1.1. If $s_{n} \rightarrow L(A)$ and $\left(s_{n}\right) \in \mathscr{S}$, then $s_{n} \rightarrow L$.

Dik, Dik and Canak [5] have generalized Theorem 1.1 by means of the concept of regularly generated sequence. Recently, Canak and Totur [2,3] have proved some tauberian theorems for which tauberian conditions are given in terms of control modulo of oscillatory behavior of a sequence.

The object of this work is to show that the proof of the main results of Section 3 below and their generalizations are essentially based on the following theorems.

Theorem 1.2. ([3]) If $s_{n} \rightarrow L(A)$ and $\left(\omega_{n}^{(m)}(s)\right)$ is $(C, 1)$ slowly oscillating for any integer $m \geq 1$, then $s_{n} \rightarrow L$.

Theorem 1.3. ([2]) If $s_{n} \rightarrow L(A)$ and $\omega_{n}^{(m)}(s) \geq-C$ for some $C \geq 0$ and for any integer $m \geq 1$, then $s_{n} \rightarrow L$

(for relevant definitions, see Section 2.)

\section{Definitions and basic properties}

Suppose throughout that $s=\left(s_{n}\right)$ is a sequence of real numbers and any term with

Received March 31, 2006.

2000 Mathematics Subject Classification. 40E05, 40G10, 40A30.

Key words and phrases. Abel summability method, regularly generated sequences, slow oscillation, general control modulo. 
a negative index is zero. For a sequence $\left(s_{n}\right)$, denote

$$
\sigma_{n}^{(m)}(s)= \begin{cases}\frac{1}{n+1} \sum_{k=0}^{n} \sigma_{k}^{(m-1)}(s)=s_{0}+\sum_{k=1}^{n} \frac{V_{k}^{(m-1)}(\Delta s)}{k}, & m \geq 1 \\ s_{n}, & m=0\end{cases}
$$

where

$$
V_{n}^{(m)}(\Delta s)= \begin{cases}\frac{1}{n+1} \sum_{k=0}^{n} V_{k}^{(m-1)}(\Delta s), & m \geq 1 \\ \frac{1}{n+1} \sum_{k=0}^{n} k \Delta s_{k}, & m=0\end{cases}
$$

and $\Delta s_{n}=\left\{\begin{array}{ll}s_{n}-s_{n-1}, & n \geq 1 \\ s_{0}, & n=0\end{array}\right.$. Note that for any integer $m \geq 0, V_{n}^{(m)}\left(\Delta \sigma^{(1)}(s)\right)=V_{n}^{(m+1)}(\Delta s)$. For the sequence $\left(s_{n}\right)$,

$$
s_{n}-\sigma_{n}^{(1)}(s)=V_{n}^{(0)}(\Delta s) .
$$

Since $\sigma_{n}^{(1)}(s)=s_{0}+\sum_{k=1}^{n} \frac{V_{k}^{(0)}(\Delta s)}{k}$, we may write (2.1) as

$$
s_{n}=V_{n}^{(0)}(\Delta s)+\sum_{k=1}^{n} \frac{V_{k}^{(0)}(\Delta s)}{k}+s_{0} .
$$

A sequence $\left(s_{n}\right)$ is said to be Abel summable to $L$, and we write $s_{n} \rightarrow L(A)$ if $\sum_{n=0}^{\infty}\left(s_{n}-s_{n-1}\right) x^{n}$ converges for $0<x<1$ and tends to $L$ as $x \rightarrow 1^{-}$. A sequence $\left(s_{n}\right)$ is said to be slowly oscillating if $\lim _{\lambda \rightarrow 1^{+}} \varlimsup_{n} \max _{n+1 \leq k \leq[\lambda n]}\left|s_{k}-s_{n}\right|=0$. A sequence $\left(s_{n}\right)$ is said to be $(C, 1)$ slowly oscillating if $\left.\underline{(\sigma}_{n}^{(1)}(s)\right)$ is slowly oscillating. A sequence $\left(s_{n}\right)$ is said to be moderately oscillating if, for $\lambda>1$, $\varlimsup_{n} \max _{n+1 \leq k \leq[\lambda n]}\left|s_{k}-s_{n}\right|<\infty$. Denote by $\mathscr{M}$ the class of moderately oscillating sequences.

It is shown in [4] that if $\left(s_{n}\right)$ is slowly oscillating, then $\left(V_{n}^{(0)}(\Delta s)\right)$ is bounded. It is clear, by (2.2), that a sequence $\left(s_{n}\right)$ is slowly oscillating if and only if $\left(V_{n}^{(0)}(\Delta s)\right)$ is bounded and slowly oscillating.

Denote by $\omega_{n}^{(0)}(s)=n \Delta s_{n}$ the classical control modulo of the oscillatory behavior of $\left(s_{n}\right)$. For each integer $m \geq 1$, define recursively $\omega_{n}^{(m)}(s)=\omega_{n}^{(m-1)}(s)-\sigma_{n}^{(1)}\left(\omega^{(m-1)}(s)\right)$, the general control modulo of the oscillatory behavior of the sequence $\left(s_{n}\right)$ of order $m$.

For each integer $m \geq 1$, all nonnegative integers $n$ and for a sequence $s=\left(s_{n}\right)$ we define inductively

$$
(n \Delta)_{0} s_{n}=s_{n}, \quad(n \Delta)_{m} s_{n}=n \Delta\left((n \Delta)_{m-1} s_{n}\right) .
$$

Lemma 2.1. ([2]) For each integer $m \geq 1, \omega_{n}^{(m)}(s)=(n \Delta)_{m} V_{n}^{(m-1)}(\Delta s)$.

Let $\mathscr{L}$ be any linear space of sequences and $\mathscr{B}$ be a subclass of $\mathscr{L}$. For each integer $m \geq 1$, define the class $\mathscr{B}^{(m)}=\left\{\left(b_{n}^{(m)}\right) \mid b_{n}^{(m)}=\sum_{k=1}^{n} \frac{b_{k}^{(m-1)}}{k}\right\}$, where $\left(b_{n}^{(0)}\right):=\left(b_{n}\right) \in \mathscr{B}$. Let $s=\left(s_{n}\right) \in \mathscr{L}$. If

$$
s_{n}=b_{n}^{(m)}+\sum_{k=1}^{n} \frac{b_{k}^{(m)}}{k}
$$


for some $b^{(m)}=\left(b_{n}^{(m)}\right) \in \mathscr{B}^{(m)}$, we say that the sequence $\left(s_{n}\right)$ is regularly generated by the sequence $\left(b_{n}^{(m)}\right)$ and $b^{(m)}$ is called a generator of $\left(s_{n}\right)$. The class of all sequences regularly generated by sequences in $\mathscr{B}^{(m)}$ is denoted by $U\left(\mathscr{B}^{(m)}\right)$.

Let $\mathscr{B}_{>}$denote the class of all sequences $b=\left(b_{n}\right)$ such that for every $\left(b_{n}\right) \in \mathscr{B}_{>}$there exists $C_{b} \geq 0$ such that $b_{n} \geq-C_{b} . U\left(\mathscr{B}_{>}^{(m)}\right)$ can be defined in the same manner as in definition above.

Let $\mathscr{B}=\mathscr{S}$. It follows from the definition that if $\left(s_{n}\right) \in U(\mathscr{S})$, then $\left(V_{n}^{(0)}(\Delta s)\right) \in U(\mathscr{S})$ and $\left(\sigma_{n}^{(1)}(s)\right) \in U\left(\mathscr{S}^{(1)}\right)$. If $\mathscr{B}$ is the class of all bounded and slowly sequences, then $U(\mathscr{B})$ is the class of all slowly oscillating sequences.

\section{Main result}

For the results in this section, we require the following lemma.

Lemma 3.1. Let $s=\left(s_{n}\right) \in \mathscr{L}$ and $k, m \geq 0$ be any integers. If $\left(V_{n}^{(k)}(\Delta s)\right) \in U\left(\mathscr{B}^{(m)}\right)$, then $(n \Delta)_{k+1} V_{n}^{(k+1)}(\Delta s)=b_{n}$.

$\left(\left(b_{n}\right)\right.$ is as in Definition in Section 2.)

Proof. If $\left(V_{n}^{(k)}(\Delta s)\right) \in U\left(\mathscr{B}^{(m)}\right)$, it then follows that

$$
V_{n}^{(k)}(\Delta s)=\sigma_{n}^{(k-1)}(s)-\sigma_{n}^{(k)}(s)=b_{n}^{(m)}+\sum_{j=1}^{n} \frac{b_{j}^{(m)}}{j}
$$

for some $\left(b_{n}^{(m)}\right) \in \mathscr{B}^{(m)}$. From (3.1), we obtain

$$
V_{n}^{(k-1)}(\Delta s)-V_{n}^{(k)}(\Delta s)=n \Delta b_{n}^{(m)}+b_{n}^{(m)} .
$$

Subtracting (3.2) from the arithmetic mean of (3.2), we have

$$
\left(V_{n}^{(k-1)}(\Delta s)-V_{n}^{(k)}(\Delta s)\right)-\left(V_{n}^{(k)}(\Delta s)-V_{n}^{(k+1)}(\Delta s)\right)=b_{n}^{(m-1)} .
$$

(3.3) can be expressed as

$$
n \Delta V_{n}^{(k)}(\Delta s)-n \Delta V_{n}^{(k+1)}(\Delta s)=b_{n}^{(m-1)},
$$

which implies $(n \Delta)_{2} V_{n}^{(k+1)}(\Delta s)=b_{n}^{(m-1)}$. By repeating the same reasoning, we have

$$
\sigma_{n}^{(1)}\left(\omega^{(k+1)}(s)\right)=(n \Delta)_{k+1} V_{n}^{(k+1)}(\Delta s)=b_{n}^{(0)}=b_{n} .
$$

Theorem 3.2. If $s_{n} \rightarrow L(A)$ and $\left(V_{n}^{(m)}(\Delta s)\right) \in U\left(\mathscr{S}^{(m)}\right)$ for any integer $m \geq 1$, then $s_{n} \rightarrow L$.

Proof. In Lemma 3.1, take $\mathscr{B}=\mathscr{S}$ and $k=m$. Then $\sigma_{n}^{(1)}\left(\omega^{(m+1)}(s)\right)=b_{n}$. If $\left(b_{n}\right) \in \mathscr{S}$, $\left(\omega^{(m+1)}(s)\right)$ is $(C, 1)$ slowly oscillating. By Theorem 1.2 , we have $s_{n} \rightarrow L$.

Theorem 3.3. If $s_{n} \rightarrow L(A)$ and $\left(V_{n}^{(m-1)}(\Delta s)\right) \in U\left(\mathscr{S}^{(m)}\right)$ for any integer $m \geq 1$, then $s_{n} \rightarrow L$. 
Proof. In Lemma 3.1, take $\mathscr{B}=\mathscr{S}$ and $k=m-1$. Then $\omega^{(m+1)}(s)=b_{n}$. If $\left(b_{n}\right) \in S$, $\left(\omega^{(m+1)}(s)\right)$ is $(C, 1)$ slowly oscillating. By Theorem 1.2 , we have $s_{n} \rightarrow L$.

Similar results can be given for one-sidedly regularly generated sequences.

Theorem 3.4. If $s_{n} \rightarrow L(A)$ and $\left(V_{n}^{(m)}(\Delta s)\right) \in U\left(\mathscr{S}_{>}^{(m)}\right)$ for any integer $m \geq 1$, then $s_{n} \rightarrow L$.

Proof. In Lemma 3.1, take $\mathscr{B}=\mathscr{S}$ and $k=m-1$. Then $\omega^{(m+1)}(s)=b_{n}$. Since $\sigma_{n}^{(1)}\left(\omega^{(m+1)}(s)\right)=$ $\omega_{n}^{(m+1)}\left(\sigma^{(1)}(s)\right)$ and $s_{n} \rightarrow L(A)$ implies $\sigma_{n}^{(1)}(s) \rightarrow L(A), \sigma_{n}^{(1)}(s) \rightarrow L$ by Theorem 1.3.

Theorem 3.5. If $s_{n} \rightarrow L(A)$ and $\left(V_{n}^{(m-1)}(\Delta s)\right) \in U\left(\mathscr{S}_{>}^{(m)}\right)$ for any integer $m \geq 1$, then $s_{n} \rightarrow L$.

Proof. In Lemma 3.1, take $\mathscr{B}=\mathscr{S}$ and $k=m-1$. Then $\omega^{(m+1)}(s)=b_{n}$. We have $s_{n} \rightarrow L$ by Theorem 1.2.

Corollary 3.6. If $\sigma_{n}^{(1)}(s) \rightarrow L(A)$ and $\left(V_{n}^{(0)}(\Delta s)\right) \in U(\mathscr{S})$, then $s_{n} \rightarrow L$.

Proof. If $\left(V_{n}^{(0)}(\Delta s)\right) \in U(\mathscr{S})$, we have $\left(V_{n}^{(1)}(\Delta s)\right) \in U\left(\mathscr{S}^{(1)}\right)$. Recalling $V_{n}^{(1)}(\Delta s)=V_{n}^{(0)}\left(\Delta \sigma^{(1)}(s)\right)$, by Theorem 3.2 we have $\sigma_{n}^{(1)}(s) \rightarrow L$, which implies $s_{n} \rightarrow L(A)$. Again by Theorem 3.2, $s_{n} \rightarrow L$.

Corollary 3.6. is Theorem 1 in [5].

For the next corollary which is Theorem 2 in [5], we need the following generalization of Theorem 1.2.

Theorem 3.7. If $\sigma_{n}^{(1)}(s) \rightarrow L(A)$ and $\left(\omega_{n}^{(m)}(s)\right)$ is $(C, 1)$ slowly oscillating for any integer $m \geq$ 1 , then $s_{n} \rightarrow L$.

Proof. It is clear that $\sigma_{n}^{(1)}\left(\omega^{(m)}(s)\right)=\omega_{n}^{(m)}\left(\sigma^{(1)}(s)\right)$. By Theorem $1.2 \sigma_{n}^{(1)}(s) \rightarrow L$, which implies $s_{n} \rightarrow L(A)$. Again by Theorem 1.2, $s_{n} \rightarrow L$.

Corollary 3.8. If $\sigma_{n}^{(1)}(s) \rightarrow L(A)$ and $\left(V_{n}^{(0)}(\Delta s)\right) \in U\left(\mathscr{M}^{(1)}\right)$, then $s_{n} \rightarrow L$. $L$.

Proof. If $\left(V_{n}^{(0)}(\Delta s)\right) \in U\left(\mathscr{M}^{(1)}\right)$, we have $\left(\sigma_{n}^{(1)}\left(\omega^{(2)}(s)\right)\right) \in \mathscr{S}$. By Theorem 3.7, we have $s_{n} \rightarrow$

Corollary 3.9. If $\sigma_{n}^{(1)}(s) \rightarrow L(A)$ and $\left(s_{n}\right) \in U\left(\mathscr{B}_{>}^{(1)}\right)$, then $s_{n} \rightarrow L$.

Proof. If $\left(s_{n}\right) \in U\left(\mathscr{B}_{>}^{(1)}\right)$, we have $s_{n}=b_{n}^{(1)}+\sum_{k=1}^{n} \frac{b_{k}^{(1)}}{k}$ for some $\left(b_{n}^{(1)}\right) \in \mathscr{B}^{(1)}$. Hence, we obtain

$$
n \Delta s_{n}=b_{n}+\sum_{k=1}^{n} \frac{b_{k}}{k} \text {. }
$$

It follows from (3.4) and (2.1) that

$$
\omega_{n}^{(1)}(s)=b_{n}=n \Delta V_{n}^{(0)}(\Delta s) \geq-C
$$

for some $C \geq 0$, which implies $\sigma_{n}^{(1)}\left(\omega^{(1)}(s)\right)=\omega_{n}^{(1)}\left(\sigma^{(1)}(s)\right)=n \Delta V_{n}^{(1)}(\Delta s) \geq-C$. Since $s_{n} \rightarrow L(A)$ implies $\sigma_{n}^{(1)}(s) \rightarrow L(A)$, by Theorem 1.3, we have $\sigma_{n}^{(1)}(s) \rightarrow L$, which implies $s_{n} \rightarrow L(A)$. Since $\omega_{n}^{(1)}(s) \geq-C$ for some $C \geq 0$, again by Theorem 1.3 , we have $s_{n} \rightarrow L$. 


\section{Acknowledgement}

The authors are grateful to the anonymous referee for his/her suggestions. This research was supported by Adnan Menderes University under the project number FEF-06011.

\section{References}

[1] İ. Canak, A proof of the generalized Littlewood Tauberian theorem, to appear in Int. J. Pure Appl. Math. Sci.

[2] İ. Canak and Ü. Totur, A Tauberian theorem with a generalized one-sided condition, Abstr. Appl. Anal., Volume 2007, 2007, Article ID 60360, 12 pages.

[3] İ. C̣anak and Ü. Totur, Tauberian theorems for Abel limitability method, Cent. Eur. J. Math. 6(2008), 301-306.

[4] M. Dik, Tauberian theorems for sequences with moderately oscillatory control moduli. Doctoral Dissertation, University of Missouri-Rolla, Missouri, 2002.

[5] M. Dik, F. Dik and İ. C̣anak, Classical and neoclassical Tauberian theorems for regularly generated sequences, Far East J. Math. Sci. 13(2004), 233-240.

[6] R. Schmidt, Über divergente folgen und lineare mittelbildungen, Math. Z. 22(1925), 89-152.

[7] Č. V. Stanojević, Analysis of Divergence: Control and Management of Divergent Process, Graduate Research Seminar Lecture Notes, edited by İ. Canak. University of Missouri-Rolla, Fall 1998.

Adnan Menderes University, Department of Mathematics, 09010, Aydin, Turkey.

E-mail:ibrahimcanak@yahoo.com

Adnan Menderes University, Department of Mathematics, 09010, Ayind, Turkey.

E-mail: utotur@adu.edu.tr 